\title{
CONFLICTO ARMADO COLOMBIANO, ESTADO MÍNIMO Y UTOPÍAS
}

\author{
BORIS SALAZAR ${ }^{1}$
}

This article applies the framework from Robert Nozick's 1974 book Anarchy, State and Utopia to the contemporary Colombian state. Nozick uses economic theory to portray the groups as competitors in a market that sells protection, and that results, counter-intuitively, in a monopoly. The article uses this theory to study the situation in Colombia, in which many people have opted out of the State, seeking protection from other groups. Indeed, there is little evidence that a traditional nation-state with sovereignty over all of its territory will arise in Colombia. But Colombians have used «filtering mechanisms» of trial and error, which Nozick theorized, to choose the best social order at the local level. Associations between communities and groups that offer protection could eventually defuse the dispute between these groups.

\section{¿Todos contra el Estado?}

Lo dijo Daniel Pécaut, en diciembre del año pasado: «En Colombia todos están contra el Estado». ¿Quiénes son todos? Pécaut (2001) no vaciló al hacer la lista de los sospechosos de siempre: «(..) los gremios empresariales, los sindicatos, los pueblos están en contra del Estado». Y si todos están en contra del Estado, ipor qué, entonces, todos, o muchos, no dejan de quejarse de la ausencia del Estado? ¿Por qué otros, incluso, piden un Estado fuerte? ¿Por qué la ilusión generalizada de superar la situación catastrófica de hoy mediante el advenimiento de un Estado que garantizara, al menos, el orden y el monopolio del uso de la fuerza? Más aún, si todos están en contra del Estado, icómo puede aún sobrevivir ese Estado maltrecho? Y esos todos, iestán formando otros Estados que pretenderían disputar el poder del

\footnotetext{
${ }^{1}$ El autor agradece los comentarios de María del Pilar Castillo.
} 
Estado bajo ataque? Y si no lo están haciendo, ¿bajo qué tipo de organización política vivirían? ¿Sería viable, de pronto, la anarquía como forma de organización política en Colombia?

Las preguntas, claro, apuntan en demasiadas direcciones. Dan la impresión de no pertenecer a un orden de investigación definido. Ni siquiera se sabe de qué Estado hablan los que así hablan del Estado ausente o del Estado fuerte por venir. Convendría cambiar el ángulo de interrogación. Preguntar, por ejemplo: iY si el Estado de hoy fuera el producto inevitable de las acciones de todos los colombianos durante el último siglo? Al hacerlo, estaría preguntando desde el enfoque que propusiera Robert Nozick en Anarquía, Estado y Utopía (1974/ 1988, de ahora en adelante AEU). Nozick llamó «explicaciones de mano invisible» (AEU, 31) al enfoque que pretendía explicar el surgimiento del Estado (sin importar su tipo) como el resultado de un proceso no planeado, un proceso cuyo «diseño completo» no ha estado en la mente de nadie. Este artículo es un intento de vincular el enfoque usado por Nozick en Anarquía, Estado y Utopía a la situación del Estado en la Colombia de hoy. No pretende afirmar la existencia de alguna similitud estructural entre el Estado a favor del cual Nozick argumentó en su libro y la situación real del Estado y el poder en Colombia. Tampoco quiere hacer una aplicación inmediata del enfoque propuesto por Nozick a la situación colombiana. Sólo quiere llamar la atención sobre la potencialidad analítica y explicativa de la propuesta de Nozick para entender lo que hoy ocurre con el Estado y con el orden político en nuestro país. Intentará argumentar a favor de la superioridad del tipo de explicación propuesto por Nozick sobre las explicaciones corrientes acerca de la ausencia del Estado, la necesidad de un Estado más fuerte o las posibilidades del Estado-Nación en las circunstancias de hoy en Colombia.

Propongo una descripción provisional de la situación del Estado en la Colombia de hoy. En un número creciente de territorios pertenecientes al dominio del Estado colombiano han aparecido grupos armados que dicen ofrecer servicios de protección a los habitantes de esas regiones (a cambio de tributación, lealtad e información), y aspiran a reemplazar al Estado en el monopolio del uso de la fuerza. Esos grupos pueden clasificarse según su número de hombres, sus reclamos históricos, su ideología, la extensión y el número de los territorios en los que operan, las redes a las que están vinculados y el número de habitantes que están bajo su protección o que deben llegar a transacciones con ellos para sobrevivir. No intentaré describir aquí ni su historia ni sus propósitos. Para efectos de esta reflexión sólo dos rasgos son relevantes: su deseo de disputar el monopolio de la fuerza que debería detentar el Estado y su oferta de protección a los civiles contra la violencia ejercida por ellos mismos y por otros grupos armados. La complejidad de las 
interacciones entre esas organizaciones armadas, el poder del Estado y las decisiones de los civiles contrasta con la liviandad de las explicaciones corrientes que sólo constatan una pretendida debilidad o ausencia del Estado y piden, en tonos diversos, su fortalecimiento, su expansión o su reemplazo por una organización estatal superior. Es mi intuición que el enfoque analítico propuesto por Nozick permitiría iniciar un acercamiento sistemático (uno de los varios posibles) a los procesos que han conducido al estado en el que hoy se encuentra el poder del Estado en Colombia².

\section{El argumento de Nozick}

¿Cuál sería el punto de partida pertinente para la reflexión propuesta? Si siguiera la tradición de la filosofía política, el punto de partida sería un Estado de Naturaleza del que se desprenderían, en forma espontánea, los procesos que han conducido al estado de hoy. Pero aquí ya aparece una dificultad. La descripción que hice más arriba de la situación colombiana habla de la existencia de varias organizaciones armadas que pretenden proteger a los ciudadanos, disputan el monopolio de la fuerza del Estado, y violan en forma sistemática los derechos de propiedad. No es el Estado de Naturaleza clásico (ni el de Locke ni el de Hobbes), pero sí es un estado en el que el poder del Estado está en cuestión y en el que en muchos territorios han surgido agencias de protección que le disputan el monopolio de la fuerza al Estado. Sería una situación a medio camino entre el Estado de Naturaleza convencional y el Estado mínimo que Nozick propone como resultado natural de la lucha entre diversas agencias de protección. Lo interesante de esta situación, lo que la llena de interrogantes, es que se produce cuando ya había un Estado en Colombia, cuando el proceso de lucha entre agencias de protección distintas ya había culminado, al parecer, con la consolidación del poder del Estado, cuando los vestigios de esa lucha habían desaparecido con el aniquilamiento de las bandas armadas a comienzos de los años sesenta, y cuando los embates de la violencia contra el Estado proveniente del narcotráfico ya habían cesado en la década pasada. A pesar de todos esos hechos, hoy nos encontramos, sin embargo, ante un proceso sui géneris

\footnotetext{
${ }^{2}$ La situación colombiana no es particular. En el llamado mundo en desarrollo muchos países se encuentran en situaciones en las que el terrorismo, el crimen organizado, la rebelión armada, la guerra civil, y la violencia sin más ponen en cuestión el monopolio del Estado sobre la fuerza. En todos ellos el poder y la viabilidad del Estado-Nación están en cuestión. El África Sub Sahariana, Latinoamérica y el Este del Asia son las regiones en las que esta situación es más pronunciada. Para una reflexión actual sobre la viabilidad del Estado tal como lo conocemos, ver: Kaplan (2002).
} 
de destrucción del Estado y de regreso hacia una situación fluida de lucha por el poder territorial y por el monopolio de la fuerza.

Una de las vías metodológicas a seguir es recorrer el camino de regreso desde el Estado-Nación hacia una situación de disputa violenta entre diversas agencias de protección. Pero, ies válido este recorrido en términos analíticos? Recuérdese que el punto de partida de Nozick es un Estado de Naturaleza a la Locke, en el que individuos libres tienen que enfrentar la violación de sus derechos por otros individuos, generando un estado de negociación interminable, motivado por los intereses egoístas de cada uno y por la necesidad, para los ofendidos, de resarcir sus pérdidas y de buscar compensación por la violencia sufrida en sus cuerpos y propiedades. Los individuos libres amenazados, dice Nozick, buscarían protegerse, primero por sí mismos, luego en unión con otros, generando un proceso evolutivo ${ }^{3}$ en el que sumarse a la fuerza creciente de los que defienden sus derechos es una estrategia superior a las otras alternativas existentes ${ }^{4}$. Sin embargo, en cada territorio, delimitado en principio en términos geográficos, varios procesos similares pueden desencadenarse al mismo tiempo. Recuérdese que se trata de múltiples procesos, iniciados en forma espontánea por diversos individuos, ninguno de los cuales posee ni su plan ni su diseño final. La implicación es transparente: «Al inicio, varias y diferentes asociaciones o compañías de protección ofrecerán sus servicios en la misma zona geográfica» (AEU, 28).

El resultado inevitable es que las agencias activas en cada territorio luchen en forma violenta hasta que una de ella alcance el monopolio de la fuerza dentro de sus límites geográficos naturales. ¿En qué radica la fuerza analítica del proceso propuesto por Nozick? En dos razones fundamentales. La primera es que demuestra la imposibilidad de la coexistencia de varias agencias de protección en un territorio. La segunda es que no hay otra línea de argumentación analítica superior (al menos hasta ese momento). Una vez establecida la existencia de múltiples agencias de protección, Nozick considera tres resultados analíticos posibles. En el primero, la lucha entre las agencias de protección lleva a la derrota sistemática de una o de varias de ellas. Los clientes de las agencias derrotadas optarán, en forma racional, por

\footnotetext{
${ }^{3}$ El Nozick de Invariances (200, 123ss), su último libro, usa con fuerza argumentos de tipo evolutivo. Es decir, un argumento se haría más fuerte si tiene de su parte un proceso evolutivo que lo fundamente.

${ }^{4}$ Hay una versión más fuerte. Jane Hampton (1986) muestra que la mejor jugada o la mejor estrategia para los individuos amenazados (o para los guerreros más débiles) en un estado de guerra es sumarse a la agencia más fuerte, así sea por la vía de la amenaza. El punto es que no hay una estrategia superior disponible, y la amenaza no es más que un incentivo para resolver el problema de coordinación existente.
} 
sumarse a la agencia vencedora - la que garantiza, en forma efectiva, su protección-. El segundo supone un estadio posterior de la confrontación entre agencias. Ahora están situadas en áreas geográficas distintas. Sin embargo, no dejarán de expandirse. Pero su expansión tiene un límite muy fuerte: en la medida en que se alejen de su centro de poder, o centro firme, la probabilidad de perder las batallas que sostienen aumenta. Los límites geográficos se ven reforzados por los límites que la interacción estratégica entre ellas genera. De nuevo, los clientes de las agencias respectivas toman decisiones racionales con respecto a su localización: o se sitúan dentro del área de influencia de su agencia inicial, o cambian de agencia de protección. El resultado, sin embargo, es el mismo: sólo una agencia de protección es viable dentro de los límites geográficos de un territorio. El tercer resultado sugiere el surgimiento de un poder superior o de unos tribunales que decidirían las disputas entre agencias que no logran un resultado definido a través de sus intercambios violentos. La predicción de Nozick es precisa: de la anarquía original, a través de múltiples procesos (que incluyen desde las economías de escala, hasta la racionalidad individual, pasando por la presión de asociaciones espontáneas), «surge algo que se parece mucho a un Estado mínimo o a un grupo de Estados mínimos geográficamente diferentes» (AEU, 29).

Nozick logra sus resultados dialogando con la teoría económica. He aquí un mercado, dice Nozick, que no puede ser competitivo en el sentido de la teoría económica contemporánea. Es decir, la interacción propia de este mercado — que Nozick no define en forma explícita- conduce a que el número de competidores se reduzca en forma dramática hasta converger a un resultado que parece contra intuitivo: un solo competidor, convertido en monopolista, permanece en él. Aunque Nozick no define en forma explícita el mercado que tiene en mente, sí define el producto que se intercambiaría en ese mercado: la protección contra otros. ¿Cuáles serían los agentes de este mercado de protección? Habría un conjunto de oferentes de protección contra otros (que incluye a todos los demás oferentes menos el mismo), y un conjunto de compradores del mismo bien. La condición para que una de las agencias logre el máximo valor para su producto depende del éxito que tenga protegiendo a sus clientes contra las amenazas de los otros, es decir, de su capacidad de aniquilar o neutralizar el poder de amenaza y de protección de sus competidores. Al contrario de lo que ocurre en los mercados competitivos, no todos los oferentes pueden vender, al precio de mercado, la cantidad que estén en capacidad de proveer del mismo producto (protección en este caso). En el mercado de protección, el valor del producto de cada agencia depende de su interacción violenta con las demás. ¿Por qué debe ser violenta esa interacción? Porque el valor del producto de cada una depende de la desvalorización del producto de las restantes, a menos que surgiera una coa- 
lición que incluyera a todas, o a la mayoría, de las agencias de protección. Si las incluyera a todas, estaríamos otra vez ante un tipo de Estado mínimo. Si al menos una quedara excluida, la coalición dominante ejercería la violencia sobre ella con resultados previsibles.

¿Qué conducta deberían seguir los compradores en este mercado particular? Deberían comprar los servicios de la agencia que los proteja, de la forma más efectiva posible, contra las amenazas de los otros individuos y de sus agencias protectoras. Pero, icómo saber con certeza cuál es la agencia más creíble? No hay una fórmula que asegure con certeza absoluta cómo elegir la agencia más efectiva de protección. Hay un camino, sin embargo: pequeñas diferencias en el número de clientes que buscan los servicios de una agencia o de otra pueden convertirse en grandes diferencias en el valor del producto protección derivado de sus acciones. La argumentación de Nozick podría presentarse así: Sea un orden del valor de los productos de protección. Este orden depende, por supuesto, de la percepción que tienen los clientes de la efectividad de los servicios prestados por cada agencia. El valor de cada producto o servicio depende, en forma estricta, del número de asociados, o de clientes, que eligen su protección. Pero este orden no es continuo: entre el valor de la agencia que tiene el mayor número de asociados y el de la segunda hay una brecha que tiende a ampliarse. Las pequeñas diferencias tienden a expandirse ${ }^{5}$, a través de la aparición de rendimientos crecientes y economías de escala, hasta hacer insostenible la situación de la segunda agencia. Al final, sólo una agencia de protección puede prestar sus servicios en un territorio geográfico.

Aquí es crucial una pregunta que Nozick no planteó en forma explícita: ¿Qué tan estable es esa configuración de Estados mínimos? Nozick supone que los límites geográficos son estructurales: ningún Estado mínimo intentaría extender sus servicios de protección más allá de los límites geográficos existentes. Pero el argumento usado para justificar el surgimiento de esos Estados puede replicarse para la interacción entre varios Estados mínimos: dos de ellos podrían aliarse contra otro y la fuerza relativa de su coalición podría hacer que otros Estados se unieran a ella, hasta formar una coalición dominante que conformaría un Estado único. Se trata de un argumento de tipo lógico que conduce, en forma inevitable, a un resultado previsible: el

\footnotetext{
${ }^{5}$ Este tipo de argumento ya había sido presentado por Hobbes (Lev, 17, 3, 85-6), tal como lo señala Hampton (169). Hobbes es quizás es el primer pensador que introduce la noción de pequeños y de grandes números en la solución de problemas de cooperación en las ciencias sociales, y el primero también que hace notar la inestabilidad inherente a las confederaciones o coaliciones pequeñas.
} 
surgimiento de una agencia dominante, o de una coalición de ellas, que se convierte en un Estado mínimo. Sin embargo, ese argumento de tipo lógico debe ser sometido a la prueba difícil de su estabilidad. Es probable que surja alguna agencia o coalición de agencias que no acepte la protección del Estado existente. Es probable que la existencia de circunstancias sociales conflictivas lleven a la violencia y al debilitamiento del monopolio de la fuerza por parte del Estado. En cualquier caso, el punto crucial es que el surgimiento de un Estado mínimo no garantiza su estabilidad. Las mismas fuerzas que llevaron a su aparición pueden llevar a su destrucción o a su fragmentación. Las vías hacia la rebelión o hacia la confrontación son múltiples. Es posible que el Estado mínimo resultante sea una agencia «ilícita», basada en el pillaje, el despojo y la extorsión. La reacción de sus protegidos no puede ser sino una: el boicot deliberado de esa agencia y de sus acciones. Es posible, también, que el Estado mínimo sea usado por algunos para su propio beneficio. La reacción de los que no participan de ese uso debe llevar a confrontar a los primeros y, en últimas, a cuestionar el poder de ese Estado. Es posible que el Estado mínimo haya sobrepasado sus límites geográficos iniciales y deba enfrentar la reacción de grupos provenientes de las regiones anexadas. Y es posible que organizaciones armadas, basadas en reclamos justos o injustos (Collier, 2000), logren crecer en ciertas regiones y llegar a convertirse en agencias que le disputan el monopolio de la fuerza al Estado. Nada garantiza, pues, que el Estado mínimo se mantenga como la agencia de protección dominante. Pareciera, entonces, que la argumentación que conduce al Estado mínimo puede conducir, también, a un estado de guerra y de confrontación entre agencias de protección.

En el caso colombiano debe considerarse otra posibilidad. ¿Qué ocurriría con la argumentación de Nozick si un Estado Nación no logra «cubrir» o controlar todos los territorios bajo su dominio? ¿Qué ocurriría si para muchos individuos la vida bajo el dominio del Estado se volviera insostenible y prefirieran emigrar hacia territorios en los que el control del Estado es inexistente? Esta última pregunta puede contestarse desde el enfoque de Nozick: los individuos que encuentran insostenible vivir bajo el control del Estado tienen todo el derecho para buscar lugares en los que puedan llevar una vida mejor, en los que incluso puedan adquirir propiedades y hacer uso pleno de las posibilidades del mercado. No estoy diciendo que las migraciones ocurridas en los años sesenta y setenta en Colombia fueron una respuesta a la opresión del Estado. Sólo estoy suponiendo que esos ciudadanos sentían que no eran protegidos por el Estado o que éste no los incluía a ellos bajo su protección o que no había oportunidades económicas suficientes en las regiones en las que vivían. La conclusión, en cualquiera de las alternativas, es que muchos ciudadanos, sin importar sus motivaciones, eligieron no perma- 
necer bajo la protección del Estado. Más que de rebelión contra el Estado, lo que aparece es un abandono voluntario de su espacio de dominio. Podría decirse que una de las características del Estado colombiano es que nunca ha tenido un dominio pleno sobre sus territorios y poblaciones. Este elemento particular del caso colombiano refuerza la idea de una disolución del Estado, mínimo o de otro tipo, a través de las acciones de individuos y grupos que no ven la aceptación del control del Estado como la mejor alternativa para sobrevivir y hacer uso de las oportunidades existentes.

¿Podría concluirse, entonces, que Nozick falló en su tarea de justificar un Estado mínimo como superior a cualquier situación de anarquía? Si su argumentación puede usarse en ambas direcciones, si el Estado mínimo surgido de su relato puede colapsar al seguir los mismos pasos lógicos de su argumentación original, ipodría declararse la derrota de su programa? Más aún: ¿podría sugerirse la futilidad de los programas filosóficos que intentan justificar la existencia del Estado? A favor de Nozick debe decirse que su preocupación fundamental era demostrar que «ningún Estado más extenso que el Estado mínimo se puede justificar» (AEU, 287). Más que justificar la existencia del Estado per se lo que interesaba a Nozick era delimitar en forma precisa lo que podría ser un Estado, que en su formación y ejercicio, no violara los derechos de los individuos. Desde ese punto de vista, vale la pena investigar las implicaciones de una situación intermedia entre el Estado de Naturaleza y el Estado mínimo. ¿Qué implicaciones analíticas puede tener una situación en la que el Estado mínimo, o un Estado-Nación más amplio, se resquebraja y los individuos deben elegir entre diversas agencias de protección? Para hacerlo, voy regresar a la situación colombiana.

\section{La utopía y el Estado mínimo}

Si, como lo sugiere Pécaut (ibidem), en Colombia muchas fuerzas sociales actúan contra el Estado, y hasta el Estado está en contra de sí mismo, valdría la pena preguntar: iA qué tipo de Estado o de organización política aspiran esas fuerzas? ¿Cuáles son sus utopías, si es que las tienen? ¿Por qué el Estado no puede convocar fuerzas suficientes para que luchen a su favor? ¿Quién está dispuesto a morir por el Estado? ¿Por qué tipo de Estado estaríamos dispuestos a luchar? Estas preguntas conducen al tratamiento que hace Nozick de las relaciones entre la utopía y el Estado mínimo. En las primeras líneas del capítulo X, Nozick interroga las relaciones precarias entre el Estado mínimo y la utopía: «Ningún Estado más extenso que el Estado mínimo se puede justificar. Pero, ino carece de lustre la idea, o el ideal, del Estado mínimo? ¿Puede llegar al corazón o inspirar a las personas para que luchen bajo su bandera?» (AEU, 287). 
Pero los individuos que estarían dispuestos a luchar, o no, por un ideal o por una utopía, no dejan de ser los sujetos racionales que fundamentan el trabajo analítico de Nozick. Es a través del filtro de la racionalidad individual como puede obtenerse algún resultado analítico con respecto a las relaciones entre utopía, Estado mínimo y legitimidad. En cada momento, tanto en el Estado de Naturaleza, como en el proceso de lucha violenta entre distintas agencias de protección, como en el ejercicio del monopolio de la fuerza por parte del Estado, lo que sostiene la dirección de las acciones de los individuos es la comparación racional que hacen entre los resultados obtenidos con la estrategia presente y los que obtendrían si siguieran una estrategia alternativa. En el lenguaje de la teoría de la elección, los individuos elegirán aquellas alternativas que les aseguran una utilidad esperada mayor. Si ellos consideran que el Estado realiza, mejor que cualquier otra alternativa existente, las tareas de protección de los ciudadanos, no tendrán ningún motivo para rebelarse o para aceptar la protección de alguna agencia o confederación alternativa. Cada uno preferirá permanecer bajo la protección del Estado actual, y no habrá ninguna alternativa que pueda competir con aquel.

Pero, ies válido definir la decisión de aceptar o no aceptar el dominio de un Estado o de un gobernante, de rebelarse o no rebelarse frente a su dominio, como proveniente de una decisión racional de los individuos? ¿Cómo podrían agregarse las decisiones de muchos individuos separados, o sólo unidos por redes precarias, hasta arribar a la gran decisión de rebelarse contra el Estado existente? Kuran ha mostrado que se requiere de unas condiciones muy especiales, entre ellas la llamada "falsificación de las preferencias», para que una rebelión tenga éxito. En el caso que nos ocupa, sin embargo, el problema es distinto. La tarea por realizar no consiste en entender, justificar y explicar una rebelión exitosa contra el poder del Estado, sino en entender el desencadenamiento y la consolidación de un conjunto de procesos distintos de rebelión (y otros que no lo son, pero que van en la misma dirección) que han conducido al debilitamiento y la erosión del poder del Estado Nación colombiano. Intentaré hacerlo desde el punto de vista del argumento de Nozick con respecto a la utopía, pero con un ancla analítica en la conducta racional de los individuos.

El hecho brutal es que en Colombia muchos individuos consideran que el Estado no garantiza la protección de sus vidas y bienes. Ya sea porque han padecido violencia por parte de otros, porque sobreviven sobre la base de aceptar contratos coercitivos de "protección» (basados en la extorsión y en la amenaza), porque conducen actividades ilegales que el Estado no puede proteger contra terceros, o porque consideran que el Estado no puede protegerlos en general contra el conjunto de amenazas existentes, muchos indi- 
viduos han tenido que considerar la posibilidad de buscar una alternativa distinta a la del Estado para proteger sus vidas y propiedades. No sé con exactitud cuántos lo consideran así, pero es claro que el crecimiento de las agencias armadas de protección, de los servicios de seguridad privada, y de las oficinas de ajuste de cuentas reflejan un vasto proceso social en marcha.

¿Son estas tendencias síntomas o señales de un proceso de rebelión contra el poder del Estado? Las organizaciones guerrilleras se llaman a sí mismas rebeldes y han intentado, en forma precaria, crear Estados primitivos en algunas regiones del país. Otras organizaciones armadas no se declaran en rebeldía contra el Estado, pero de hecho lo suplantan en su tarea básica de protección de los ciudadanos, y llegan incluso a incursionar en el campo de la redistribución del ingreso agenciando proyectos locales de reforma agraria. Pero más importante aún que estas manifestaciones organizadas, y en armas, del proceso de cerco al Estado, es el conjunto de procesos espontáneos que, a través de la violencia, del crimen organizado, de la depredación cotidiana y estratégica, han reducido todavía más el papel del Estado como garante de la vida y de la propiedad de los ciudadanos. Más allá de si nos encontramos o no ante un proceso de rebelión generalizado, el punto decisivo es que las cuestiones de vida o muerte para los colombianos ya no pasan por el Estado. En una situación normal ningún ciudadano tendría que pensar en el problema de la protección de su vida y de sus intereses. Le bastaría con saber que la convención social que le dio ese poder al Estado sigue en pie. Es más: no tendría ni siquiera que saberlo o pensarlo porque no habría motivo para hacerlo. En la situación colombiana ocurre lo contrario: cada uno tiene que tomar decisiones con respecto a su seguridad y a la protección de su vida y propiedades. Cada ciudadano debe considerar ahora las alternativas existentes y tratar de encontrar el mejor curso de acción en condiciones muy difíciles. El cálculo racional (aunque limitado) de los resultados de las alternativas disponibles se pone de nuevo al orden del día. La pregunta que surge es: ¿Qué tipo de orden podría surgir de esa multiplicidad de cálculos racionales ocurriendo en forma simultánea?

La forma en que he presentado mi argumento podría llevar a pensar que sólo en tiempos recientes, quizás demasiado recientes, el poder del Estado y su monopolio sobre el uso de la fuerza ha sido puesto en cuestión. No es así, por supuesto. Más allá de las muchas guerras civiles y de la violencia generalizada que cualquiera puede constatar en nuestra historia, el poder del Estado en Colombia ha sido atravesado por la acción, durante muchos años, de los partidos liberal y conservador y, más tarde, por las múltiples organizaciones que han entrado en la disputa del uso de la fuerza. Esa acción ha tenido un carácter local y regional, con agentes muy definidos: el gamonal, el jefe de vereda, el jefe político regional, el jefe nacional de directorio. Sus 
acciones han contado con el concurso de redes sociales que usan y se apropian de los fondos del Estado, distribuyen sus nóminas, administran justicia, adjudican propiedades, y manipulan a las Fuerzas Armadas y de Policía. En cada región, en cada municipio y vereda, el Estado tomaba (y aún hoy lo hace) el color y el rostro del partido o de la facción dominante en cada momento ${ }^{6}$. La imagen del gobernante o del Estado único, cuya fuerza y poder hace que todos los ciudadanos no tengan por qué pensar, en términos racionales, en la búsqueda de otras alternativas de protección no hace parte de nuestra historia política. Abusando un poco de los términos, podría decirse que una convención social ha surgido como dominante: aquella que hace irracional confiar en el poder de protección del Estado y vuelve racional el buscar otras alternativas de protección. Se trata de una situación fluida y compleja en la que el Estado no es sino una más entre las varias agencias de protección que se disputan territorios y poblaciones en Colombia. Es la que cuenta con mayores recursos, militares y económicos, pero es también la más frágil. Desde muchos puntos, diversas organizaciones, grupos e individuos depredan sus recursos y exigen respuestas puntuales que, en general, el Estado no puede producir. En otras palabras, resulta muy costoso para el Estado comportarse como Estado en todo el territorio del país. Si el Estado fuera otro agente racional, a la Nozick, debería enfrentar el problema de elegir qué acciones merecen respuesta inmediata, qué regiones son decisivas para mantener su poder, qué territorios requieren de una mayor inversión en fuerzas ofensivas y disuasivas, qué territorios, en cambio, pueden esperar. La igualdad en la entrega de protección no es una virtud de un Estado reducido y amenazado.

Si el Estado enfrenta un proceso de reducción de su monopolio de la fuerza y de su control territorial, y algunas organizaciones armadas ilegales pretenden convertirse en Estados primitivos en algunas regiones del país, ¿qué tipo de orden podría surgir de una situación tan compleja? ¿Qué lugar habría en él para la utopía que Nozick propusiera en el último capítulo de su libro? Nótese que el ideal de un Estado Nación único, cuyo poder volviese inútil a cualquier otra agencia de protección, no ha podido realizarse en Colombia. Nótese, también, que en la situación de hoy, tanto el Estado, como las organizaciones armadas ilegales, tienen en mente objetivos nacionales. Cada uno, a su manera, pretende cubrir todo el territorio nacional y proteger a todos los ciudadanos que en él viven. Esas aspiraciones, sin embargo, no están

${ }^{6}$ Carlos Miguel Ortiz (1985) lo ha documentado de manera extensa para el caso del Quindío de la Violencia clásica. Malcom Deas (1995) ha defendido la tesis del origen político del conflicto colombiano, basándose en la forma en que la tradición partidista colombiana definió bandos, territorios y comportamientos. 
fundamentadas ni en sus acciones reales ni en los efectos de esas acciones. Cada agencia de protección puede reclamar, hasta cierto punto, ciertos territorios, la lealtad de parte de la población en algunas regiones, la convergencia entre sus propuestas y la utopía particular de algunos individuos y de algunas comunidades. Pero ninguna en particular puede reclamar la movilización de la vasta mayoría de los ciudadanos alrededor del tipo de comunidad que propone, y de su monopolio sobre la protección y la fuerza. Una vasta distancia se abre entre las aspiraciones absolutas y nacionales de las agencias de protección que hoy se disputan el control estatal en Colombia y sus posibilidades reales.

Dice Nozick que en el marco para la utopía cabrán todas las utopías posibles:

la utopía consistirá en utopías, en muchas comunidades diversas y divergentes en las cuales las personas llevan diferentes clases de vida bajo diferentes instituciones. Algunas clases de comunidades serán para la mayoría más atractivas que otras; las comunidades crecerán y decaerán (AEU, 300).

¿Qué implicaciones puede tener esta formulación para la situación colombiana? Podría decirse que la inestabilidad de nuestras convenciones sociales, la fragilidad del Estado, el avance y la proliferación de instituciones espontáneas indican la dinámica de muchos individuos y comunidades que, en diversas formas, y persiguiendo distintas utopías, o aun sin perseguirlas, han buscado, durante años, arreglos sociales alternativos. Si nos atuviéramos a la vigencia de procesos sociales espontáneos, o de mano invisible, esa búsqueda, iniciada desde muchos lugares, y desde perspectivas divergentes, no ha logrado converger, al menos, hacia un Estado mínimo en el sentido de Nozick. Sugiero que tomemos esa consideración como un tipo de evidencia provisional con respecto a los procesos reales que han venido ocurriendo. Es decir, si las explicaciones de mano invisible fueran pertinentes, la evidencia que surge de la realidad colombiana indicaría que no hay nada que permita esperar el surgimiento y la consolidación de un Estado Nación tradicional con poder soberano sobre todo el territorio nacional. No es lo que ha ocurrido y no es probable que ocurra en el futuro.

Desde una perspectiva optimista, la aparente anarquía de hoy podría interpretarse como una búsqueda intensa y múltiple de arreglos sociales diversos, en un contexto restringido por la guerra y la depredación. No diré, por supuesto, que múltiples utopías se disputan su supervivencia en un marco ideal para la utopía. Ni la guerra, ni la extorsión ni la depredación generalizada permiten imaginarlo. Pero tanto la guerra, como la depredación alertan acerca de la futilidad de buscar una utopía única, un sistema privilegiado al que convergerían todos los individuos y todas las comunidades, a pesar de haber sostenido y soñado utopías diferentes. Es la situación en la que nos encontramos hoy. Ninguna de las guerras que hoy se libran en Colombia permiten pensar, o prometen, aun a los más optimistas, que un sistema social único surgirá de sus cenizas y nos conducirá a un mundo posible cercano a la utopía de todos. 
¿Qué se puede esperar de la interacción local entre utopías diversas? ¿Qué se puede esperar de las asociaciones estables que pueden surgir, y están surgiendo, de los procesos sociales ocurridos en los últimos años? ¿Acaso surgirá una confederación de Estados mínimos surgida del impacto de múltiples interacciones locales? En su capítulo sobre la utopía, Nozick recurre a la teoría de juegos cooperativa de $n$ personas para modelar los procesos que conducen a asociaciones estables. Suponiendo una racionalidad colectiva mínima, Nozick sugiere unas reglas sencillas para la formación de asociaciones estables en un contexto de total libertad para las utopías. La primera establece que todo individuo está en libertad de mantener la utopía que desee y de elegir el mundo posible, o la asociación, más cercana a la utopía que imagina. La segunda dice que ningún individuo abandonará la asociación en la que se encuentra, si no hay ninguna otra en la que obtenga una valoración mayor a la que recibe hoy por su contribución marginal. Pero, como lo dice el propio Nozick (AEU, 294), estas reglas implican una explicación circular y los intentos realizados en la teoría de juegos, hasta ese momento, no habían logrado una solución definitiva del problema.

Queda lo que Nozick llama los mecanismos de filtración: los individuos, a través de la interacción social, ensayarían diversas forma de organización social, intentarían distintas utopías, y seleccionarían aquellas que resistieran mejor la crítica proveniente de la experiencia real. Este tipo de mecanismo de filtro se puede encontrar en Hayek (1960), y en la visión evolutiva que Popper (1972) propuso del proceso de avance del conocimiento. En ambas propuestas nadie sabe lo que surgirá más adelante, pero todos saben que en el proceso todas las visiones y todas las utopías han sido ensayadas y pueden, incluso, ensayarse de nuevo, a pesar de haber sido rechazadas o superadas en ocasiones anteriores. Quizás la feroz disputa entre distintas agencias de protección en la que hoy vivimos pueda comenzar a desarmarse a partir de las interacciones locales entre agencias de protección y comunidades. En muchas regiones de la Colombia de hoy, individuos y comunidades intentan alcanzar algún tipo de asociación estable, olvidando las pretensiones nacionales y la idea de imponer algún tipo de sistema único o de utopía absoluta. Las asociaciones y arreglos sociales que surgen de esas interacciones son un paso adelante con respecto al Estado de Naturaleza y son superiores, también, con respecto a la aplicación mecánica de unos principios definidos, que nunca podrán captar la diversidad de situaciones que pueden surgir en el futuro.

Pero este proceso de interacción local no es independiente del desarrollo de la guerra. Será inevitable que en ese proceso ideal de ensayo y error en la búsqueda de arreglos sociales alternativos, las comunidades deban enfrentar el problema de las coaliciones con las agencias de protección que se disputan el control de territorios y poblaciones. El punto de partida es la extrema desigualdad de las partes en términos de su poder militar. Las agen- 
cias de protección — cualesquiera que sea la realidad de sus accionescuentan con el poder de las armas y de la organización militar. Las comunidades que no han aceptado la protección de una agencia o del Estado, no lo tienen. Aquí aparece una objeción crucial al mecanismo de filtro y de interacción local: si las agencias de protección tienen objetivos nacionales y buscan la imposición de sistemas absolutos, icómo pueden las comunidades aceptar su protección? Y si no la aceptan, icómo pueden negociar en pie de igualdad sin entrar en algún tipo de coalición con alguna o algunas de ellas? ¿Cómo podría conseguirse que todas las partes aceptaran una negociación basada en la exclusión de la violencia organizada como forma de coacción política y de apropiación económica? Las respuestas a estas preguntas no pueden encontrarse en la filosofía política. Deben encontrarse en la evolución de la guerra y en el camino que conduzca hacia un Estado mínimo o hacia una configuración de ellos.

\section{Bibliografía}

Collier, P. 2000. «Economic Causes of Civil Conflict and their Implications for Policy». Washington, DC: World Bank, Mimeo.

Hampton, J. Hobbes and the Social Contract Tradition. Cambridge: Cambridge University Press.

Hayek, F. A. 1960. The Constitution of Liberty. Chicago: Chicago University Press.

Kaplan, R.D. La anarquía que viene. Barcelona: Ediciones B.

Kuran, T. 1993. «Sparks and prairie fires: A theory of unanticipated political revolution», in Witt, U. (ed.), Evolutionary Economics. Aldershot: Edward Elgar.

Nozick, R. 2001. Invariances. The Structure of the Objective World. Cambridge, MA: Harvard University Press.

Nozick, R. 1974/1988. Anarquía, Estado y Utopía. México DF: Fondo de Cultura Económica.

Pécaut, D. 2001. «En Colombia todos están contra el Estado». Semana, diciembre 10, 34-5.

Popper, K. 1972. Objective Knowledge. Oxford: Oxford University Press. 